identifying the key measures of policy impact. We aimed to develop a set of logic models that could be widely used in tobacco control policy evaluation.

Methods We developed logic models for policies recently implemented in England, including smokefree legislation, changes to age of sale laws and point-of-sale display bans. We used an iterative process to develop models for each policy, before combining outcomes into a single overarching model. We initially reviewed policy documents to identify the outcomes expected to result from the implementation of each policy, and then conducted a literature review of existing policy evaluations to identify further outcomes. The models were refined through research team meetings, and revised according to feedback from a range of stakeholders including a public involvement group and national tobacco control policymakers. Results The final models represented expected causal pathways for each policy and identified the populations in which outcomes were expected to occur. The models included shortterm outcomes (such as policy awareness, compliance and social cognitive outcomes), intermediate outcomes (such as changes in smoking behaviour) and long-term outcomes (such as mortality, morbidity and health service usage).

Conclusion The logic models guided the development of hypotheses and choice of outcome measures in subsequent evaluations of tobacco control policies. The use of logic models enables prospective and theory-based planning of evaluation analyses, which in turn enhances the transparency of policy evaluation. The use of logic models should be encouraged in the evaluation of tobacco control policy, as well as in other areas of public health.

This study is funded by NIHR PRP (PR-R14-1215-24001). The views expressed are those of the authors and not necessarily those of the NHS, the NIHR, the Department of Health and Social Care, arms length bodies or other government department.

\section{OP91 PRICE RESPONSIVENESS OF CIGARETTE CONSUMPTION AMONG PAKISTANI ADULTS: EVIDENCE FROM GLOBAL ADULT TOBACCO USE SURVEY (GATS)}

H Masud*, 0 Oyebode. Warwick Medical School, University of Warwick, Coventry, UK

10.1136/jech-2019-SSMabstracts.95

Background Raising tobacco taxation and prices is an effective policy tool to reduce tobacco use and has been identified by the WHO Framework Convention for Tobacco Control (FCTC) as a leading tobacco control strategy, and highlighted with the sustainable developmental goals (SDGs).

It is important to base the tobacco taxation and pricing policies on the consumers' actual responsiveness to price rises which is measured by price elasticity (PE) of demand. Policy makers use PE estimates to design effective taxation policies and to project the impact of different policy options on tobacco consumption and their revenues.

This study aims to estimate the PE of demand for cigarettes among adult Pakistanis and to distinguish the effect on smoking prevalence and intensity.

Methods We used data from Pakistan's 2014 Global Adult Tobacco Survey (GATS). GATS is part of the WHO's global tobacco surveillance system to monitor tobacco use and key tobacco control indicators in a nationally representative household survey. A total of 7831 individuals aged $15+$ years participated in the survey. We estimated PE using the standard two-part model for cross-sectional studies; smoking participation and smoking intensity. The probability of smoking cigarettes was estimated using a logit model, and for smoking intensite an Ordinary Least Squares regression model was used. Explanatory variables in both models were: price, demographic characteristics, indicators of the socioeconomic status of individuals, rural/urban residence, knowledge about smoking hazards, exposure to anti-smoking messages and cigarette advertisements and smoking restrictions at home. Analyses were weighted to adjust for national representation.

Results The adult PE of cigarettes demand was estimated to be -0.43 . The overall PE is comprised of a statistically nonsignificant PE of smoking participation $(-0.17)$ and statistically significant $\mathrm{PE}$ of smoking intensity $(-0.26)$, indicating that a $10 \%$ increase in price is expected to reduce smoking prevalence by $1.7 \%$ on average and can decrease the average number of cigarettes smoked by $2.6 \%$.

The price elasticity is slightly reduced if estimated for only males $(-0.40)$ and the magnitude increased to -0.71 if the highest income quintile is excluded from the analysis. If individuals exposed to high price cigarettes (PKR >150) are excluded from the study, an increase in the price of (low priced) cigarettes would significantly decrease both smoking participation and intensity and impact would be higher for smoking participation ( $\mathrm{PE}=-1.01)$.

Conclusion The analysis yielded negative price elasticity of cigarettes demand for Pakistani adults, indicating an increase in price would decrease cigarette use both by decreasing smoking prevalence and daily consumption among smokers in the country. We found that an increase in the price of low priced cigarettes would have a greater negative impact on smoking prevalence and daily consumption by smokers. These findings demonstrate that cigarette prices can be used as an effective policy tool to control smoking in Pakistan.

\section{Population Health}

\section{OP92 USE OF SYSTEMATIC REVIEW EVIDENCE BY PUBLIC HEALTH PROFESSIONALS IN ENGLISH LOCAL AUTHORITIES: A QUALITATIVE STUDY}

E South*, T Lorenc. Centre for Reviews and Dissemination, University of York, York, UK

\subsection{6/jech-2019-SSMabstracts.96}

Background Studies have generally found limited use of academic research in policy-making. In 2013 the context for public health decision-making in England changed from the NHS to local authorities (LAs). This represents a different policy environment; however, public health is still considered an evidence-based profession. Despite systematic reviews being regarded as the best available evidence for health decisionmaking, little is known about how they are used in local government. As the relationship between evidence and policy is potentially complex, it is important to consider the role of research beyond directly informing decisions. The aim of this study was to explore the extent to which public health decision-makers in LAs engage with systematic review evidence and how they do so.

Methods Semi-structured interviews were conducted with senior public health professionals involved in decision-making in Yorkshire and the Humber LAs. Sampling was purposive 